\title{
Improved IEEE 802.11 Point Coordination Function Considering Fiber-Delay Difference in Distributed Antenna Systems
}

\author{
Yuting Fan ${ }^{1,2}$, Jianqiang $\mathrm{Li}^{1}$, Kun $\mathrm{Xu}^{1 *}$, Nathan J. Gomes ${ }^{2}$, Xun $\mathrm{Lu}^{1}$,Yitang Dai ${ }^{1}$, Feifei Yin ${ }^{1}$ \\ ${ }^{1}$ State Key Laboratory of Information Photonics and Optical Communications, Beijing University of Posts and \\ Telecommunications, Beijing, 100876, P. R. China \\ Email: xukun@bupt.edu.cn \\ ${ }^{2}$ Broadband and Wireless Communications Group, School of Engineering and Digital Arts, University of Kent, Kent, UK \\ Email: N.J.Gomes@kent.ac.uk
}

\begin{abstract}
In this paper, we present an improved IEEE 802.11 wireless local-area network (WLAN) medium access control (MAC) mechanism for simulcast radio-over-fiber-based distributed antenna systems where multiple remote antenna units (RAUs) are connected to one access point (AP). In the improved mechanism, the fiber delay between RAUs and central unit is taken into account in a modification to the conventional point coordination function (PCF) that achieves coordination by a centralized algorithm. Simulation results show that the improved PCF outperforms the distributed coordination function (DCF) in both the basic-access and request/clear-to-send modes in terms of the total throughput and the fairness among RAUs.
\end{abstract}

Keywords- Distributed antenna system, IEEE 802.11, point coordination function, radio-over-fiber.

\section{INTRODUCTION}

Distributed antenna systems (DASs) using radio-over-fiber (RoF) links have been demonstrated as a commonly used infrastructure solution to provide broadband wireless coverage within a geographic area with reduced total power and improved reliability [1-2]. In a RoF-based DAS, multiple spatially separated remote antenna units (RAUs) are fiberconnected to a center unit where base station or access point facilities are placed. It achieves the broadband communications with the features of low attenuation, large capacity, small-size RAUs and centralized management, compared with the current scheme where multiple small WLANs cover the certain area. In some DAS applications, a single set of base station facilities in the center unit is connected to multiple RAUs to extend the indoor wireless coverage of the base station and to share the hardware and bandwidth resource. This kind of DAS is often called a simulcast DAS architecture, where a single base station simultaneously broadcasts radio-frequency (RF) signals to multiple RAUs in the downlink. In the uplink, the user stations covered by different RAUs share transmission medium and base station/access point (AP) facilities. For cost-effective inbuilding coverage in practice, a great number of wireless service providers or carriers around the world have deployed simulcast DAS in buildings and public venues for improving the quality and coverage of radio systems based on IEEE
Worldwide Interoperability for Microwave Access (WIMAX), and $3^{\text {rd }}$ Generation Partnership Project $(3 G)$ and long-term evolution (LTE) standards. With the shared RoF-based DAS infrastructure, the IEEE 802.11-compliant wireless local-area network (WLAN) signals may also be distributed in the same buildings and public venues as an important complement to the mobile network signals, such as LTE.

The typical architecture of a simulcast WLAN-over-fiber DAS is shown in Fig. 1. In the downlink, multiple RAUs distributed in different locations receive the optical signals carrying the WLAN signals split from one source AP in the central unit over different-length fibers. A reverse process occurs in the uplink. User stations accessing the AP through different RAUs have to contend for the WLAN channel with multiple medium access control (MAC) mechanisms, in the presence of different fiber delay.

IEEE 802.11 standards define two MAC mechanisms [3]: Distributed Coordination Function (DCF) and Point Coordination Function (PCF). DCF, which has been implemented in most of the WLAN products, is a random channel-access technique based on carrier sense multiple access with collision avoidance (CSMA/CA), where each user station has a right to initiate its transmission and decides the proper transmitting occasion itself. DCF commonly has two working modes: the basic access mode and the Request to Send/Clear to Send (RTS/CTS) mode. Many previous works have studied IEEE 802.11 DCF both in the basic access mode and the RTS/CTS mode in the context of point-to-point RoF systems, where a dedicated RAU is assumed to be connected to a WLAN AP [4-6]. They explored the MAC timingconstraint problems induced by fiber delay and identified some upper bounds in certain scenarios. Recently, we have investigated the throughput performance of DCF in simulcast RoF-based DASs [7]. An analytical model was derived to quickly evaluate the throughput performance in the presence of both the inter-RAU hidden nodes (HN) problems and fiberdelay difference. Regarding the PCF mechanism, previous studies mainly focused on the performance in traditional WLAN (non-RoF) [8-10]. The most different character of the $\mathrm{PCF}$ is that it achieves coordination by a centralized algorithm 


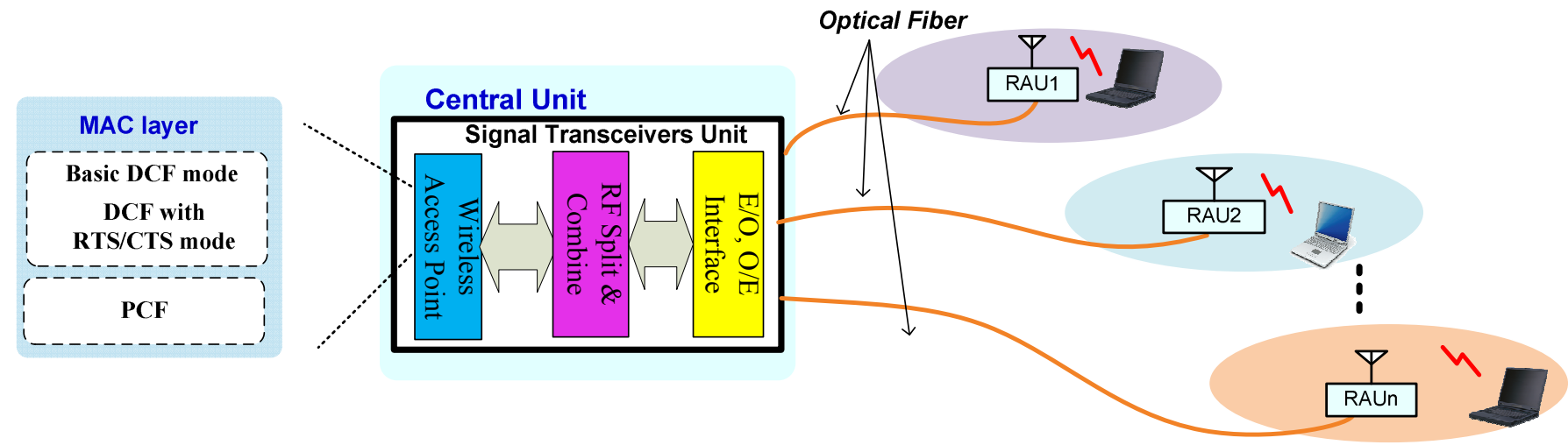

Fig.1 The architecture of a simulcast RoF-based WLAN distributed antenna system

where the AP runs the algorithm, while the DCF has a distributed algorithm run by all the stations.

For other popular mobile or wireless standards such as WIMAX, LTE etc., the tolerance to the inserted fiber propagation delay is found to be much greater than with the 802.11 protocol when they are employed in RoF-DAS systems [11-14]. These mobile systems are designed for greater wireless distances, and hence tolerate some delay variation, but also employ MAC protocols which are somewhat more centralized, at least after some initial contention procedure for users to gain access. No inter-RAU HN problems beset WIMAX- or LTE-RoF-DAS due to the lack of contention, and little effect from fiber-length differences between the base station and different RAUs exists when carefully arranging

\section{OVERVIEW OF TWO IEEE 802.11 MAC MECHANISMS}

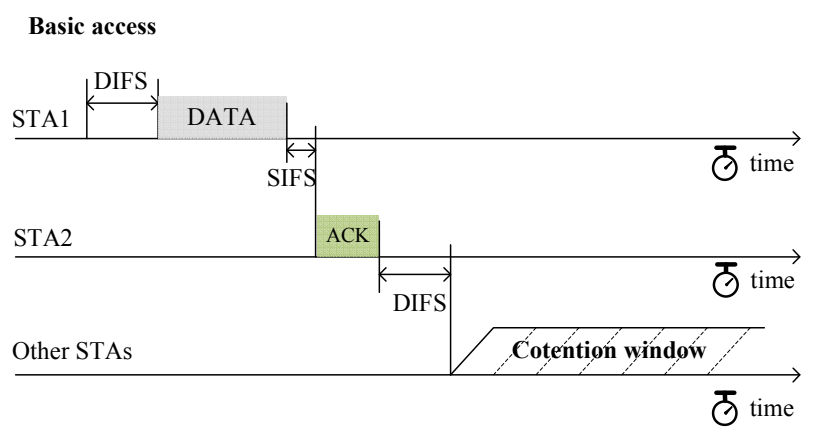

(a)

Fig. 2 A timing diagram for the basic access DCF mode

As Fig. 2 depicts, the basic access DCF is a random channel-access scheme based on CSMA/CA. Each station has a right to initiate its transmission after the channel has been idle for a DCF interframe space (DIFS) interval, following exponential backoff time rules. On winning the contention, the station gains the chance to transmit a single MAC Service Data Unit (MSDU) on the channel. After a successful transmission, the destination station waits for a short interframe space (SIFS) interval and then sends an ACK packet to confirm the correct and adjusting related parameters such as the Transmit/Receive Transition Gap (TTG) and Cyclic Prefix (CP) durations. In 802.11-over-fiber DASs, the centralized characteristic of PCF inspires us to investigate its performance in simulcast RoFbased DASs. It is supposed to be a promising solution for the inter-RAU HN problems and may decrease the fiber-length difference effect.

In this paper, we first investigate the performance of the polling-based PCF in the simulcast RoF-based DASs and present an improved PCF algorithm considering fiber-delay difference effect. In our mothod, an adaptive PCF Interframe Space (PIFS) parameter is used to adapt to the different fiber delays. Simulations are conducted using OPNET Modeler 17.5.

reception of data in the MAC layer. Once a packet is transmitted, the source station starts a countdown timer, which is known as an ACK timeout. When employing the basic access DCF in simulcast RoF-DASs, the stations covered by one RAU cannot, in general, perceive whether a station covered by other RAUs has occupied the channel. Therefore, the inter-RAU HN problems are serious, especially when the network load is high.

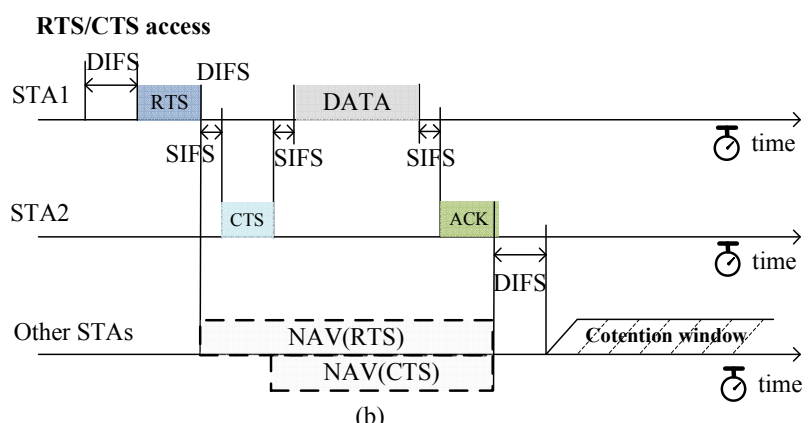

Fig. 3 A timing diagram for DCF in the RTS/CTS mode

As shown in Fig. 3, DCF in RTS/CTS mode with virtual sensing is a promising method to prevent $\mathrm{HN}$ problems in 
traditional WLANs and has been proved effective in WLANover-fiber systems [6-7]. The source station sends a RTS frame to check the availability of the destination in typical CSMA/CA style firstly. When the destination receiver receives the RTS frame, it will then reply with a CTS frame to avoid simultaneous transmissions of data. On hearing the exchange of RTS/CTS frames, the other stations will set their network allocation vector (NAV) to certain times during which the stations need to defer before the next contention interval. After the exchange of RTS/CTS frames, the procedure performed for data exchange is similar to that used in the basic-access DCF, except SIFS are employed. The RTS threshold parameter indicates a specific packet size above which the RTS/CTS mode is employed; otherwise the Basic Access mode is employed. When employing the DCF RTS/CTS mode in simulcast RoF-DASs, the serious inter-RAU HN problems can be alleviated to some extent [6-7]. However, in the DCF RTS/CTS mode, the MAC layer overhead is larger compared to that in Basic Access DCF, and the tolerance to the fiber delay differences remains problematic.

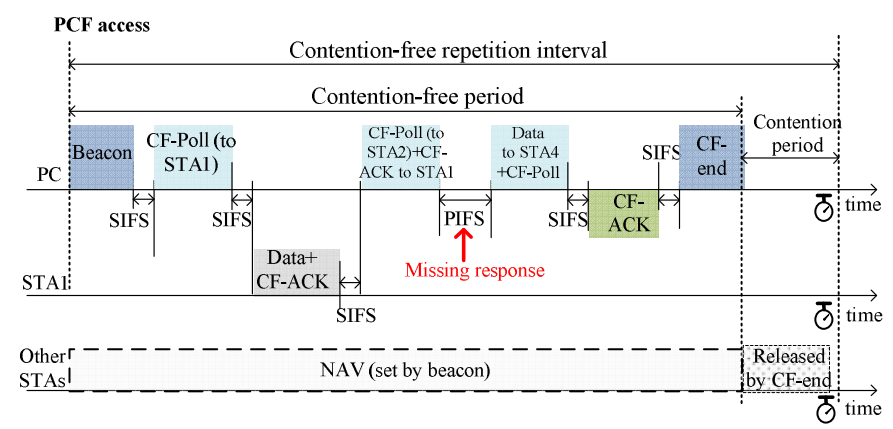

Fig. 4 A timing diagram for PCF

For PCF, all the stations in the polling list are polled by a Point Coordinator (PC) normally situated in the AP. The PCF uses a centralized resource scheduler, where a single AP controls the associated stations' access to the channel by sending the polling messages. If the AP has data to send to the station, the data can be included in the polling messages. PCF is usually used to provide priority to some specific services or users. It may be specifically used for real-time applications. With its strong characteristics of a centralized allocation scheduler, we want to use it to improve the throughput performance in a simulcast RoF-DAS, where the stations covered in different located RAUs can barely sense each other properly. A strong demand for a centralized allocation arises because of the needs of the blind stations.

As shown in Fig. 4, the time window is divided into two parts, the contention-free period (CFP) in which PCF is used and contention period (CP) in which DCF is used. The contention-free repetition interval determines the frequency with which the PCF occurs. It begins with a beacon frame and ends with a CF-end frame or a CF-end + ACK frame. The beacon frame includes the parameter of CFP maximum duration which indicates the maximum size of the CFP. Stations receive the beacon frame and set their NAV to the CFP maximum duration. In the CFP, only the station which receives the polling frame from the $\mathrm{PC}$ can transmit a packet. If the PC doesn't receive a CF-ACK, it will poll the next station after the PCF interframe space (PIFS), denoted as $T_{P I F S}$. In our studies, we found that the typical PIFS $(19 \mu$ s for $802.11 \mathrm{~g})$ in traditional WLANs is too short to adapt to the fiber-fed networks; the PC may not receive the CF-ACK due to the round-trip fiber delay $F_{\text {delay }}(1 \mathrm{~km}$ fiber results in $10 \mu$ s delay in round trip delay). Hence, we present an adaptive method, adding the round-trip fiber delay in the PIFS $T_{P I F S \text {-ada }}$ to ensure that the $\mathrm{PC}$ can receive the $\mathrm{CF}_{-} \mathrm{ACK}$ and not be affected by the delays. It can be defined as: $T_{P I F S-a d a}=T_{P I F S}+F_{\text {delay }}$.

\section{ThroughPUT PERFORMANCE IN SIMULCAST RoF- BASED DAS WITH DIFFERENT-LENGTH FIBER LINKS}

First, we assume identical fiber length between central unit and RAUs ( $1 \mathrm{~km}$ and $10 \mathrm{~km}$ are studied). The packet size in our analyses is always 1500 bytes and RTS threshold is 500bytes, which means that all the packets are transmitted with a prior RTS/CTS exchange when the RTS/CTS mode is employed. We also assume a saturated load during the simulation period, which means that there is always a packet in the buffer waiting for transmission after a current successful transmission. Then, we run simulations to evaluate the performance of different MAC mechanisms as a function of the number of RAUs in the simulcast architecture.

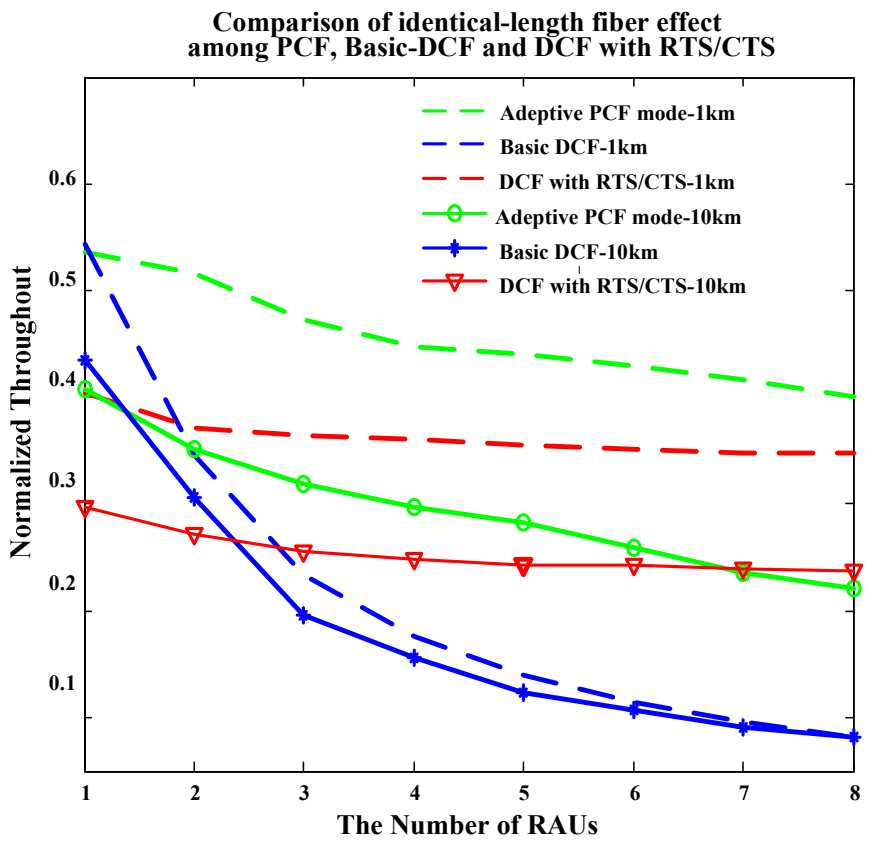

Fig. 5 Normalized throughputs for the basic-access DCF mode, DCF in RTS/CTS mode and adaptive PCF as a function of the number of RAUs assuming identical fiber length

As observed from Fig. 5, the proposed improved PCF outperforms DCF in both Basic Access and RTS/CTS modes, which proves the prediction that the adaptive PCF can avoid the inter-RAU HN problems through its centralized polling mechanism, especially over shorter length fiber links. We are using normalized throughput as a performance indicator and we normalized the results by the maximum data rate $54 \mathrm{Mb} / \mathrm{s}$, which we used in our simulation. Additionally, the trends of the adaptive PCF and DCF RTS/CTS performance both 
decrease smoothly and slowly with increased number of RAUs. However, an improvement of $25.7 \%$, which is the ratio of average improvement to the average throughput of RTS/CTS mode, can be observed in $1 \mathrm{~km}$-length situation and $15.72 \%$ in $10 \mathrm{~km}$-fiber-length situation, which shows the lesser overhead of the adaptive PCF than the DCF RTS/CTS mode. The adaptive PCF is more efficient than the DCF RTS/CTS mode. But, the advantage of the adaptive PCF over DCF becomes weaker as the fiber length increase.

Second, we investigate the performance of different MAC mechanisms in the presence of fiber-delay difference with the following assumptions:

(1) All the stations enable the PCF function in the MAC layer;

(2) A dual-RAU scenario is considered in the simulations for convenience, and a single station accesses the AP in each RAU;

(3) One RAU (in this case, RAU-A) is connected to the central unit with fixed $1 \mathrm{~km}$ fiber link, while a variable length $F$ (from $1 \mathrm{~km}$ to $19 \mathrm{~km}$ ) is assigned to the other RAU (RAUB).

(4) The same packet size (1500bytes), RTS threshold (500bytes) as the former analysis are employed as is a saturated load.

As the PIFS is a global parameter, the adaptive PIFS value $T_{\text {PIFS-ada }}$ has to take into account the longest fiber delay in the RoF-based DAS. For example, if one RAU is connected to the AP with a $1 \mathrm{~km}$ fiber link, while another RAU employs a $10 \mathrm{~km}$ fiber link to connect to the AP, the $T_{P I F S-a d a}$ should be set to $\left(T_{P I F S}+2 \times 10 \mathrm{~km} \times 5 \mu \mathrm{s}\right)$ to ensure that every station in the DAS can be polled in a period sufficient to obtain the CF-ACK for the poll, although more time is wasted and the throughput is expected to decrease a little.

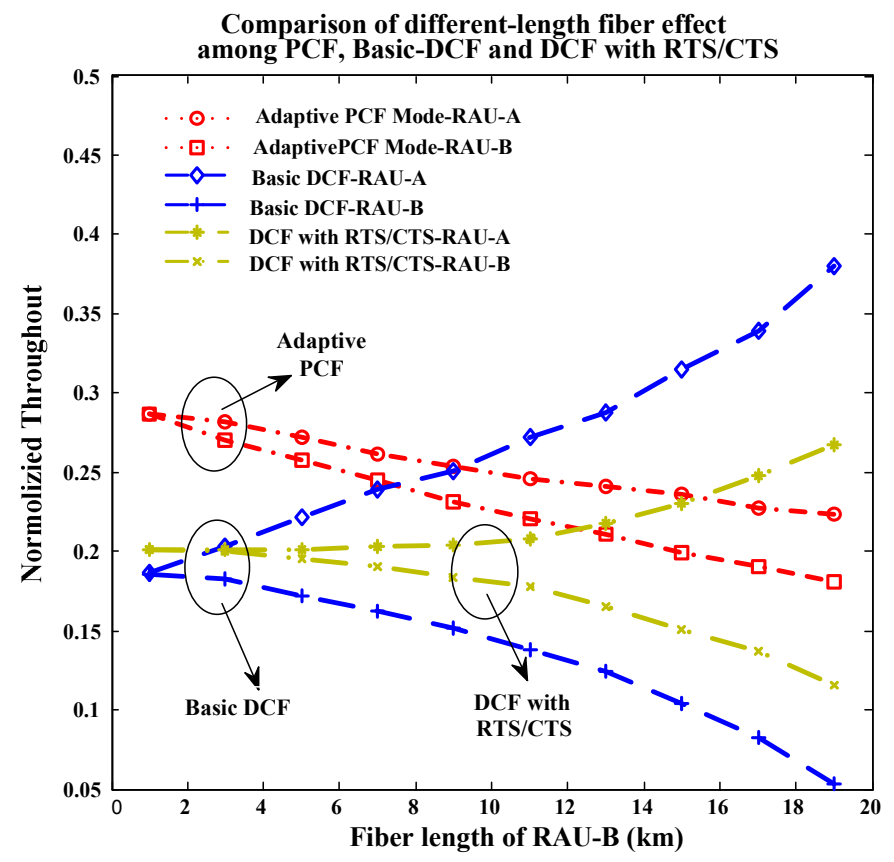

Fig. 6 Throughput performance of each RAU as the function the length of one of the fiber links assuming different fiber lengths in a two-RAU scenario.
Fig. 6 depicts the throughput performance of each RAU as the function of the fiber length between the AP and RAU-B. Although the overall throughput slightly drops for each RAU as the fiber length of RAU-B increases, a much smaller performance deviation between the two RAUs can be observed in the adaptive PCF mode, which indicates that the adaptive PCF provides more fairness to all the stations covered by every RAU in the presence of fiber length differences. In this case, the fairness means that the probabilities of the stations in each RAU to access the channel are almost the same, which can be reflected at the difference between the two RAUs' throughputs. Hence, the adaptive PCF can effectively prevent the situation where the stations are unable to access to the channel through the RAU with a long fiber link. The major reason behind this is that the centralized polling scheme for PCF solves the interRAU HN problems. In this paper, we assumed a saturated load and a packet size which is the largest size used in Ethernet. The results show a good throughput performance for adaptive PCF. We can see that the adaptive PCF is a promising solution to be employed in a simulcast RoF-DAS to improve the throughput performance and enhance the fairness to all the users.

\section{CONCLUSION}

In this paper, we introduced the PCF in simulcast RoFbased DASs. In the presence of fiber-delay difference among RAUs, an adaptive PCF mechanism was proposed by considering the delay induced by the longest fiber link. The adaptive PCF and DCFs in basic-access and RTS/CTS modes were compared with the help of simulations using OPNET. It is shown that the adaptive PCF is a promising mechanism for simulcast RoF-DASs in terms of the overall throughput and fairness among RAUs.

\section{ACKNOWLEDGMENT}

This work was supported in part by National 973 Program (2012CB315705), NSFC Program (61271042, 61107058, 61302086, and 61302016), Specialized Research Fund for the Doctoral Program of Higher Education (20130005120007), Program for New Century Excellent Talents in University (NCET-13-0682), and Fundamental Research Funds for the Central Universities. The authors are grateful to Dr Majlinda Mjeku for useful advice on the insertion of fiber delays in the OPNET modelling. The author, Miss Yuting Fan is presently working at University of Kent, which is supported by China Scholarship Council (CSC).

\section{REFERENCES}

[1] M. J. Crisp, S. Li; A. Wonfor, R. V. Penty and I. H. White, "Demonstration of a Radio over Fibre Distributed Antenna Network for Combined In-building WLAN and 3G Coverage," in OFC/NFOEC 2007. Conference on, pp. 1-3, 2007.

[2] J. Z. Wang, H. L. Zhu and N. J. Gomes, "Distributed Antenna Systems for Mobile Communications in High Speed Trains," Selected Areas in Communications, IEEE Journal on, vol. 30, pp. 675-683, 2012.

[3] Part 11: Wireless LAN Medium Access Control (MAC) and Physical Layer (PHY) Specifications, IEEE Standard 802.11, Mar. 2012.

[4] B. Kalantari-Sabet, M. Mjeku and N. J. Gomes, "Performance Impairments in Single-Mode Radio-Over-Fiber Systems Due to MAC Constraints," Journal of Lightwave Technology, vol. 26, pp. 2540-2548, 2008 . 
[5] S. Deronne, V. Moeyaert, and S. Bette, "Analysis of the MAC performances in $802.11 \mathrm{~g}$ radio-over-fiber systems," 18th IEEE Symposium on in Communications and Vehicular Technology(SCVT), in the Benelux, 2011.

[6] M. Mjeku and N. J. Gomes, "Analysis of the request to send/clear to send exchange in WLAN over fiber networks," Journal of Lightwave Technology, vol.26(15), 2531-2539, 2008.

[7] Y. T. Fan, J. Q. Li, K. Xu, et al., "Performance analysis for IEEE 802.11 distributed coordination function in radio-over-fiber-based distributed antenna systems," Optics Express, vol. 21, pp. 20529-20543, Sep 2013.

[8] K. H. Chou and W. Lin, "Performance Analysis of Packet Aggregation for IEEE 802.11 PCF MAC-Based Wireless Networks," IEEE Transactions on Wireless Communications, vol. 12, pp. 1441-1447, 2013.

[9] Q. L. Liu, D. F. Zhao, and H. W. Ding, "An Improved Polling Scheme for PCF MAC Protocol," 7th International Conference on Wireless Communications, Networking and Mobile Computing (WiCOM), 2011.
[10] B. Sikdar, "An analytic model for the delay in IEEE 802.11 PCF MACbased wireless networks," IEEE Transactions on Wireless Communications, vol. 6, pp. 1542-1550, 2007.

[11] P. Sklikas, M. Mjeku and N. J. Gomes, "MAC layer performance evaluation of IEEE 802.16e Radio-over-Fiber networks," IEEE Topical Meeting on Microwave Photonics (MWP), pp. 350-353, 2010.

[12] P.H. Gomes, N.L.S. da Fonseca, O.C. Branquinho, "Analysis of performance degradation in Radio-Over-Fibre systems based on IEEE 802.16 protocol", IEEE Latin-American Conference on Communications, LATINCOM '09, Sept. 2009.

[13] B. L. Dang, M. Garcia Larrode, R. Venkatesha Prasad, I. Niemegeers, A. M. J. Koonen, "Radio-over-fiber based architecture for seamless wireless indoor communication in the $60 \mathrm{GHz}$ band", Comp. Commun., vol. 30, pp. 3598-3613, Dec. 2007.

[14] P. Sklikas and N. J. Gomes, "Downlink-uplink timing synchronization and optical multipath limitations in 3GPP TD-LTE Radio-over-Fiber networks," International Topical Meeting on Microwave Photonics (MWP), pp. 212-215, 2012. 